\title{
EFEITO DA APLICAÇÃO DE HERBICIDA HORMONAL EM DIFERENTES ESTÁDIOS DE DESENVOLVIMENTO DE TRIGO (Triticum aestivum L. Cvs. Embrapa 16 e BR 23)
}

\author{
Osmar Rodrigues ${ }^{1}$, José A. Marchese ${ }^{2}$, Leandro Vargas ${ }^{3}$, José A. O. Velloso ${ }^{4}$ e Rita \\ de C. S. Rodrigues ${ }^{5}$ \\ 1 Pesquisador da Embrapa Trigo, Caixa Postal 451, 99001-970 Passo Fundo, RS. E-mail: osmar \\ @cnpt.embrapa.br \\ 2 Eng. Agrôn. CEFET-PR - Centro Federal de Educação Tecnológica do Paraná, Caixa Postal 571, CEP 85503- \\ 390 Pato Branco, PR. \\ 3 Pesquisador da Embrapa Trigo, Caixa Postal 451, 99001-970 Passo Fundo, RS. \\ 4 Eng. Agrôn., Caixa Postal 451, 99001-970 Passo Fundo, RS. \\ 5 Eng ${ }^{a}$ Agrôn. Caixa Postal 451, 99001-970 Passo Fundo, RS. Bolsista da FAPERGS.
}

\begin{abstract}
RESUMO - A fitotoxidade dos herbicidas hormonais a base de reguladores de crescimento em trigo está associada ao estádio de desenvolvimento do meristema apical da planta. Aplicações entre os estádios de duplo anel e espigueta terminal são improváveis de causarem deformações nas espigas e danos na produção de grãos. Assim, torna-se necessária a correta determinação desses estádios para um uso seguro desses herbicidas. Mesmo assim, pequenas alterações podem ocorrer em função do ambiente, do padrão de translocação do herbicida na planta e do desenvolvimento diferencial dos afilhos na planta. Com o objetivo de conhecer o comportamento de duas cultivares de trigo (Embrapa 16 e Trigo BR 23), frente a aplicação de herbicida hormonal, foram realizado dois experimento à campo. Aplicações de herbicidas hormonais entre os estádios de duplo anel e espigueta terminal não causaram deformações nas plantas nem danos na produção de grãos. Contudo, aplicações anteriores a esse período em ambas as cultivares, causaram deformações nas espigas e na folha bandeira, sem no entanto afetarem o rendimento de grãos. Por outro lado aplicações após esse período, causaram reduções significativas no rendimento em ambas as cultivares, reduzindo em até 60 $\%$ o rendimento de grãos. O número de grãos por espiga foi o componente determinante da redução do rendimento em ambas as cultivares.
\end{abstract}

Palavras-chave: Triticum aestivum (L.), Estádios de cresimento, Herbicidas hormonais e Épocas de aplicação.

\section{EFFECT OF GROWTH REGULATOR HERBICIDES APPLIED AT DIFFERENT STAGES OF DEVELOPMENT IN WHEAT}

ABSTRACT -The phytotoxicity level caused by hormonal herbicides that work as growth regulators in wheat plants is associated with the growth stage of the apical meristem. Applications of such herbicides when wheat plants are at double ridge and at terminal spikelet stages are very unlikely to cause damage to the ear and to the grain production. So, it is necessary to determine precisely the stages of plant development for having a secure use of these herbicides. However, small alterations may occur as a function of the environmental conditions, the mode of translocation of the herbicides, and tiller development. So, to determine the effect of the application of hormonal herbicide in two wheat cultivars (Embrapa 18 and BR 23), two field 
experiments were established. The application of hormonal herbicides at double ridge and at terminal spikelet stages did not cause any damage to the ears or to grain production. Applications before such stages caused deformities in the ears, flag leaves, and did no harm to grain yield. Applications after such stages reduced grain yield significantly for both cultivars, reaching the level of $60 \%$ reduction. The number of grains per ear was the component that mostly reduced grain yield for both cultivars.

Key-words: Triticum aestivum (L.), developmental stages, hormonal herbicides, timing of application.

\section{INTRODUÇÃO}

A sensibilidade das plantas a herbicidas hormonais a base de reguladores de crescimento é grandemente determinada pelo estádio de desenvolvimento do meristema apical. O desenvolvimento da planta de trigo pode ser definido como uma seqüência de mudanças morfológicas (Fenologia) e/ou função de alguns órgãos, modulado por fatores de ambiente. Essa seqüência de mudanças são influenciadas diferencialmente por fatores adversos do ambiente (secas, excesso de água, temperaturas elevadas, deficiência nutricional etc..), tornando-se de fundamental importância a identificação das diferentes fases de desenvolvimento e suas necessidades/sensibilidades. Para caracterizar o desenvolvimento da planta de trigo, tem sido proposto dois tipos de critérios: 1)baseado nas mudanças na morfologia externa da planta e 2)no grau de evolução do ápice de crescimento da planta.

O ápice de crescimento (órgão gerador de folhas e/ou de espiguetas) se encontra localizado abaixo da superfície do solo até o momento em que começa o crescimento do colmo (final do afilhamento). Nesse período, em que se encontra abaixo da superfície do solo gera folhas até o momento do aparecimento do duplo anel (primeira espigueta) e, a partir daí, gera espiguetas até o aparecimento da espigueta terminal (Início do alongamento). A partir do estádio de espigueta terminal, em cada espigueta no ápice de crescimento localizado no último entrenó do colmo, inicia-se a diferenciação dos órgãos das flores. Após, a espiga começa seu crescimento e na seqüência sua extrusão. Depois da sua extrusão e quando todos órgãos das flores já estão formados, ocorre a fecundação(Antese).

A duração das diferentes fases do desenvolvimento da planta de trigo, é controlada por vários fatores: temperatura, disponibilidade hídrica, vernalização, fotoperíodo e características genética do germoplasma.

A aplicação de herbicidas hormonais pode provocar alterações morfofisiológicas, condicionadas a fase de desenvolvimento que se encontra a planta, não necessariamente com reflexos negativos na produção de grãos. Assim, como não poderia deixar de ser, o uso de herbicidas hormonais esta condicionado ao estádio de desenvolvimento da planta no momento da aplicação, onde a tolerância ao herbicida é maior. Leaden \& Lozano,(1986)estudando cultivares de trigo de ciclo curto e longo na Argentina observaram que a tolerância a herbicidas hormonais aumentou a partir do estádio de duplo anel, atingindo o seu máximo no estádio de espigueta terminal (Início do alongamento). Assim, a identificação correta dos estádios, independente do tempo cronológico, torna-se imprescindível. Mesmo 
assim, alterações podem ocorrer, por efeito do ambiente (Andersen,1954), no padrão de translocação do herbicida nas plantas e pelo desenvolvimento diferencial dos afilhos (Friesen \& Olson,1953).

Aplicações desses herbicidas é uma prática muito corrente no meio agrícola, contudo as indicações do momento correto de sua aplicação ainda oferecem muita incerteza, aja vista o grande número de problemas de fitotoxidez observado ano a ano. Problemas dessa natureza podem ser oriundos de dificuldades na identificação do momento de maior tolerância das plantas aos referidos herbicidas. Aplicações muito precoces causam deformações em folhas, espiguetas opostas, espiguetas faltantes, aristas retorcidas, alargamento do ráquis e redução de estatura da planta (Friesen et al, 1963; Sharman,1978; Leaden \& Lozano, 2004), não necessariamente associada com redução do rendimento. Contudo, Tottman ,(1977) observou reduções no rendimento com aplicações precoces de 2,4-D e MCPA e mal formação de espigas, por interferência nos primórdios de espiguetas no ápice de crescimento. Por outro lado, aplicações tardias (após o estádio de Espigueta Terminal), causam reduções no rendimento de grãos , o que tem sido atribuído a interferência dos herbicidas na esporogênese (Dercheid,1951; Pinthus \& Natowitz,1967; Leaden \& Lozano,2004).

Em condições de campo, a recomendação para utilização de herbicidas hormonais em trigo esta baseada em características externas da planta como número de folhas e número de afilhos ou fases Fenológicas como afilhamento/primeiro nó, como período mais tolerante (REUNIÃO DA COMISSÃO SUL-BRASILEIRA DE PESQUISA DE TRIGO - Recomendações,1996). Apesar disso, essas características tem sido apontadas como pouco precisa para determinar o grau de desenvolvimento do ápice de crescimento (Tottman,1977; Tottman \& Duval,1978; Lozano et al.,1984) Além disso, o pleno afilhamento ou início do alongamento são características que apresentam dificuldades para sua determinação a campo, além de não refletirem o grau de desenvolvimento do ápice de crescimento. Assim, alguns critérios de identificação baseado em características externas (Feekes,1941; Large,1954; Zadoks et al.,1974), parecem não serem muito adequados. Tentativas tem sido realizadas no sentido de melhorar o grau de precisão na identificação de estádios chaves do desenvolvimento de cereais (Tottman,1976; Tottman \& Makepeace, 1979). Nesse sentido, Tottman (1978), propõe a utilização da altura da bainha no colmo principal, como critério para aplicação de herbicidas hormonais, por refletir melhor o grau de desenvolvimento do ápice. Lozano et al., (1984), observaram que o estádio de duplo anel coincidiu com $5 \mathrm{~cm}$ de altura da bainha para as cultivares de ciclo longo e de $3,5 \mathrm{a} \mathrm{4,0} \mathrm{cm}$ para as cultivares de ciclo intermediário e curto, respectivamente. Nerson et al.,1980, complementam os aspectos externos da planta de trigo com estudos sobre os estádios de desenvolvimento do ápice de crescimento e propõem uma escala de desenvolvimento, na qual 0; 1; 2 (corresponde ao estádio vegetativo), 3 (duplo anel) e 9 (espigueta terminal).

A utilização correta dos herbicidas hormonais implica no reconhecimento das fases de desenvolvimento da planta de trigo bem como a influência dos fatores de ambiente sobre a duração das mesmas e o comportamento diferencial de cultivares. Nesse sentido, o conhecimento do comportamento de novos cultivares com germoplasma mexicano e com diferentes hábitos de crescimento, torna-se imprescindível. 
O objetivo do presente trabalho foi estudar os efeitos do herbicida hormonal (Dicamba), aplicado em diferentes estádios de desenvolvimento do ápice de crescimento de dois cultivares de trigo, sobre o rendimento de grãos e seus componentes.

\section{MATERIAIS E MÉTODOS}

O estudo foi realizado no ano agrícola de 1992 e 1993, no campo experimental da Embrapa Trigo, Passo Fundo, RS em solo LVE, com as seguintes características: em $1992\left(\mathrm{pH}_{\mathrm{H} 20}=5,4 ; \mathrm{pH}_{\mathrm{SMP}}=5,8 ; \mathrm{Al}^{3+}=0,33 \mathrm{me} / 100 \mathrm{~g} ; \mathrm{Ca}^{2+}=5,80\right.$ $\mathrm{me/100g} ; \mathrm{Mg}^{2+}=2,72 \mathrm{me} / 100 \mathrm{~g} ; \mathrm{P}=18,6 \mathrm{ppm} ; \mathrm{K}=130 \mathrm{ppm} ;$ e $\left.\mathrm{MO}=2,3 \%\right)$ em $1993\left(\mathrm{pH}_{\mathrm{H} 20}=\right.$ 5,$6 ; \mathrm{pH}_{\mathrm{SMP}}=6,0 \quad ; \mathrm{Al}^{3+}=0,03 \mathrm{me} / 100 \mathrm{~g} ; \mathrm{Ca}^{2+}=5,21 \mathrm{me} / 100 \mathrm{~g} ; \mathrm{Mg}^{2+}=2,28 \mathrm{me} / 100 \mathrm{~g} ; \mathrm{P}=16,0$ ppm; $\mathrm{K}=112 \mathrm{ppm}$ e $\mathrm{MO}=2,2 \%$. A adubação com NPK foi efetuada antes da semeadura, conforme recomendação da Comissão Sul-brasileira de Pesquisa de trigo, utilizando-se 250 e $300 \mathrm{~kg} / \mathrm{ha}$ de adubo na formulação 5-20-20, em 1992 e 1993 respectivamente. No estádio de duplo anel e espigueta terminal, em cada ano, foram realizadas aplicações de $25 \mathrm{~kg} / \mathrm{ha}$ de nitrogênio em cobertura, utilizando-se uréia como fonte. O material vegetal utilizado foi constituído por duas cultivares de trigo: Trigo BR 23 (Precoce) e Embrapa 16 ( Precoce com pequena exigência de vernalização), submetidas a 14 tratamentos, constituídos de aplicações semanais de herbicida hormonal. Em 1992, utilizou-se apenas a cultivar de trigo BR 23, já em 1993 as duas cultivares fizeram parte do estudo, cujas datas de semeadura e emergência encontram-se na Tabela 01 . Na Tabela 02, encontram-se os dados meteorológicos ocorridos durante o período de desenvolvimento da cultura, nos respectivos anos.

Durante o ciclo da cultura, foram realizadas duas aplicações de fungicidas para controle de doenças da parte aérea. Na primeira aplicação utilizou-se $0,5 \mathrm{l} / \mathrm{ha}$ de Tilt ( propiconazole $125 \mathrm{~g}$. i.a/ha ) e na segunda utilizou-se uma mistura de 0,5 I/ha de Tilt $+0,75$ l/ha de Folicur (Tebuconazole 187 g.i.a/ha).

Os tratamentos, foram os seguintes: $\mathrm{T} 1=$ Testemunha absoluta (Capina mecânica) ; T2 a T14 foram compostos de aplicações semanais do herbicida. O herbicida utilizado foi dicamba $(480 \mathrm{~g} / \mathrm{l})$ na dosagem de $0,3 \mathrm{l} / \mathrm{ha}$, utilizando-se pulverizador com pressão constante ( $\mathrm{CO}_{2}$ comprimido), bico Teejet 11002 e 200 I/ha de volume de solução. A semeadura foi realizada mecanicamente em parcelas experimentais constituídas de 9 linhas de $5 \mathrm{~m}$ de comprimento, com densidade de 300 sementes aptas por metro quadrado. Mudanças na morfologia externa das plantas (Zadoks et al.,1974) e no ápice de crescimento (Nerson et al.,1980), foram acompanhadas periodicamente antes da aplicação de cada tratamento. Para tal, coletou-se 5 plantas por repetição onde foram dissecadas e avaliadas o grau de desenvolvimento do ápice de crescimento, com utilização de uma lupa binocular. Após a colheita final, avaliou-se o rendimento de grãos, número de espigas $/ \mathrm{m}^{2}$, número de grãos $/ \mathrm{m}^{2}$, número de grãos/espigas e peso de mil grãos.

O delineamento experimental foi o de blocos ao acaso, com quatro repetições. Os dados obtidos foram submetidos à análise de variância. O F-teste foi usado ao nível de $5 \%$ de probabilidade e as diferenças entre as médias foram comparadas pelo teste de Duncan $(p>0,05)$. 
Tabela 01 - Datas de semeadura e emergência para as cultivares de trigo Embrapa 16 e BR 23 cultivadas em 1993 e 1992-1993, respectivamente.

\begin{tabular}{llcc}
\hline Cultivares & Estádios & Ano 1992 & Ano 1993 \\
\hline Br 23 & Semeadura & $13 / 07$ & $20 / 07$ \\
& Emergência & $27 / 07$ & $30 / 07$ \\
Embrapa 16 & Semeadura & - & $22 / 06$ \\
& Emergência & - & $02 / 07$ \\
\hline
\end{tabular}

Tabela 02 - Valores de Insolação, temperaturas médias, temperaturas máximas e temperaturas mínimas e precipitação pluviométrica mensal.

\begin{tabular}{|c|c|c|c|c|c|c|c|c|}
\hline \multicolumn{2}{|c|}{ Parâmetros Anos } & Jun & Jul & Ago & Set & Out & Nov & Dez \\
\hline $\begin{array}{r}\text { Insolação } \\
\text { (Horas) }\end{array}$ & $\begin{array}{l}1992 \\
1993\end{array}$ & $\begin{array}{l}132,1 \\
153,8\end{array}$ & $\begin{array}{l}139,4 \\
141,2\end{array}$ & $\begin{array}{l}162,4 \\
190,7\end{array}$ & $\begin{array}{l}186,4 \\
145,6\end{array}$ & $\begin{array}{l}171,3 \\
185,3\end{array}$ & $\begin{array}{l}231,3 \\
222,4\end{array}$ & $\begin{array}{l}281,7 \\
270,5\end{array}$ \\
\hline $\begin{array}{l}\text { Tmax. } \\
\left({ }^{\circ} \mathrm{C}\right)\end{array}$ & $\begin{array}{l}1992 \\
1993\end{array}$ & $\begin{array}{l}20,3 \\
18,2\end{array}$ & $\begin{array}{l}15,8 \\
17,1\end{array}$ & $\begin{array}{l}18,0 \\
21,2\end{array}$ & $\begin{array}{l}21,2 \\
19,8\end{array}$ & $\begin{array}{l}23,9 \\
25,2\end{array}$ & $\begin{array}{l}25,6 \\
26,2\end{array}$ & $\begin{array}{l}28,3 \\
27,8\end{array}$ \\
\hline $\begin{array}{l}\text { Tmin. } \\
\left({ }^{\circ} \mathrm{C}\right)\end{array}$ & $\begin{array}{l}1992 \\
1993\end{array}$ & $\begin{array}{c}11,3 \\
8,8\end{array}$ & $\begin{array}{l}6,5 \\
8,2\end{array}$ & $\begin{array}{l}8,3 \\
9,0\end{array}$ & $\begin{array}{l}10,9 \\
10,2\end{array}$ & $\begin{array}{l}12,8 \\
15,0\end{array}$ & $\begin{array}{l}13,7 \\
15,0\end{array}$ & $\begin{array}{l}16,8 \\
17,0\end{array}$ \\
\hline $\begin{array}{l}\text { Pluvios. } \\
\qquad(\mathrm{mm})\end{array}$ & $\begin{array}{l}1992 \\
1993\end{array}$ & $\begin{array}{l}109,9 \\
137,2\end{array}$ & $\begin{array}{l}241,6 \\
284,0\end{array}$ & $\begin{array}{r}147,8 \\
15,2\end{array}$ & $\begin{array}{l}186,3 \\
136,5\end{array}$ & $\begin{array}{l}136,5 \\
154,2\end{array}$ & $\begin{array}{l}280,2 \\
273,9\end{array}$ & $\begin{array}{l}130,3 \\
259,0\end{array}$ \\
\hline
\end{tabular}

\section{RESULTADO E DISCUSSÃO}

O rendimento de grãos, considerando os resultados obtidos em $1992 \mathrm{com}$ a cultivar BR 23 , evidencio que a aplicação do herbicida quando realizada no estádio vegetativo (anterior ao estádio de duplo anel) até próximo ao estádio 55 (Zadoks et al.,1974) não causou reduções significativas no rendimento de grãos. Contudo , causaram mal formações nas espigas e folhas. Resultados semelhantes foram observados por Ivany \& Nass (1984). Por outro lado, quando a aplicação foi realizada no estádio 55 (Zadoks et al.,1974) e próxima a antese, ocorreram reduções de aproximadamente $60 \%$ no rendimento de grãos, estatisticamente significativas (Tabela 3). A causa de tais reduções, tem sido atribuída ao efeito dos herbicidas hormonais na esporogênese causando esterilidade das flores (Dercheid,1951; Pinthus \& Natowitz,1967). Tais resultados, foram confirmados em 1993 com as cultivares de trigo BR 23 e Embrapa 16 (Tabelas 4 e 5).

A tolerância ao herbicida aplicado aumentou a partir do estádio de duplo anel, atingindo um máximo no estádio de espigueta terminal. Resultados semelhantes foram observados por Tottman (1977), onde aplicações precoces de 2,4-D e MCPA causam alterações morfológicas nas folhas e espigas por interferir na disposição de novas folhas e primórdios de espiguetas no ápice do colmo. Leaden \& Lozano (1986), utilizando uma mistura entre 2,4-D éster e picloran observaram redução no rendimento de grãos quando a aplicação foi realizada posterior ao período de 
tolerância, e que esta redução foi mais importante do que aplicação anterior a esse período.

Tabela 03 - Aplicação de herbicida hormonal em diferentes estádios de crescimento de trigo (Trigo BR 23) no rendimento de grãos e seus componentes, em 1992.

\begin{tabular}{|c|c|c|c|c|c|c|c|}
\hline Datas & deEstádios de & № $\quad$ de & № & Peso & eGrãos/ & Rendimen & de grãos \\
\hline Aplicação & Cresc. & Espigas $/ \mathrm{m}^{2}$ & Grãos $/ \mathrm{m}^{2}$ & 1000 grãos & Espiga & Kg/há & \% Redução \\
\hline $\begin{array}{ll}\text { T1 } & \text { (Test }\end{array}$ & & 336,2 & $8287 a$ & $35,0 \mathrm{bc}$ & $25,2 a b c$ & $3345 a$ & 0,0 \\
\hline T2 $(11 / 08$ & $01^{*}$ & 296,8 & $7875 a$ & 35,2 bc & $26,6 a b c$ & $3187 \mathrm{a}$ & 4,7 \\
\hline T3 (18/08 & $02^{*}$ & 284,8 & $7527 \mathrm{a}$ & $34,7 \quad \mathrm{c}$ & $26,8 \mathrm{ab}$ & $3004 a b$ & 0,2 \\
\hline T4 $(25 / 08)$ & 8) $03-04^{*} \mathrm{DA}$ & 329,3 & $7838 a$ & $34,4 \mathrm{~cd}$ & $24,3 a b c$ & $3086 a$ & 7,7 \\
\hline T5 $(01 / 09)$ & $05-06^{*}$ & 310,7 & 7913 a & $34,1 \mathrm{~cd}$ & $25,6 a b c$ & $3108 a$ & 7,1 \\
\hline T6 $(08 / 09)$ & 9) $07-08^{*} \mathrm{ET}$ & 294,9 & $7917 \mathrm{a}$ & $35,0 \mathrm{bc}$ & $27,0 \mathrm{ab}$ & $3173 a$ & 5,1 \\
\hline T7 $(15 / 09)$ & $\square 09^{*}$ & 283,0 & $7830 a$ & $34,9 \mathrm{bc}$ & $27,7 \mathrm{a}$ & $3125 a$ & 6,6 \\
\hline T8 (22/09) & & 344,9 & $7755 a$ & $34,2 \mathrm{~cd}$ & $22,6 \mathrm{bcd}$ & $3047 a b$ & 8,9 \\
\hline T9 $(29 / 09)$ & & 317,3 & $5849 \mathrm{~b}$ & $37,4 a b$ & $18,4 d$ & $2515 b$ & 24,8 \\
\hline T10 (6/10) & $55^{* *}$ & 311,8 & $2900 \mathrm{c}$ & $36,5 \mathrm{bc}$ & 9,3 e & $1215 c$ & 63,7 \\
\hline $\mathrm{T} 11(13 / 10)$ & & 313,7 & $2754 c$ & $39,4 a$ & $9,1 \mathrm{e}$ & $1269 \mathrm{c}$ & 62,1 \\
\hline T12(20/10) & $10.5-3^{* *}$ & 314,7 & $8281 \mathrm{a}$ & $35,3 \mathrm{bc}$ & $26,3 a b c$ & $3356 \mathrm{a}$ & 0,0 \\
\hline $\mathrm{T} 13(27 / 10)$ & & 397,9 & $8156 a$ & 31,9 de & $21,9 \mathrm{~cd}$ & $2977 a b$ & 12,0 \\
\hline $\mathrm{T} 14(05 / 11)$ & & 330,8 & $8435 a$ & $31,5 \mathrm{e}$ & $25,4 a b c$ & $3047 a b$ & 8,9 \\
\hline$\overline{\mathrm{CV}}(\%)$ & & 17,33 & 12,42 & 5,23 & 14,63 & 13,54 & \\
\hline 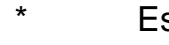 & & olvime & (Nerso & 880) & & & \\
\hline$* *$ & dir do & ento $(Z a$ & locks et & 974) & & & \\
\hline$\star \star \star *$ & Tra & doc nol & & & & & \\
\hline
\end{tabular}

Tabela 04 - Aplicação de herbicida hormonal em diferentes estádios de crescimento de trigo (Trigo BR 23) no rendimento de grãos e seus componentes, em 1993.

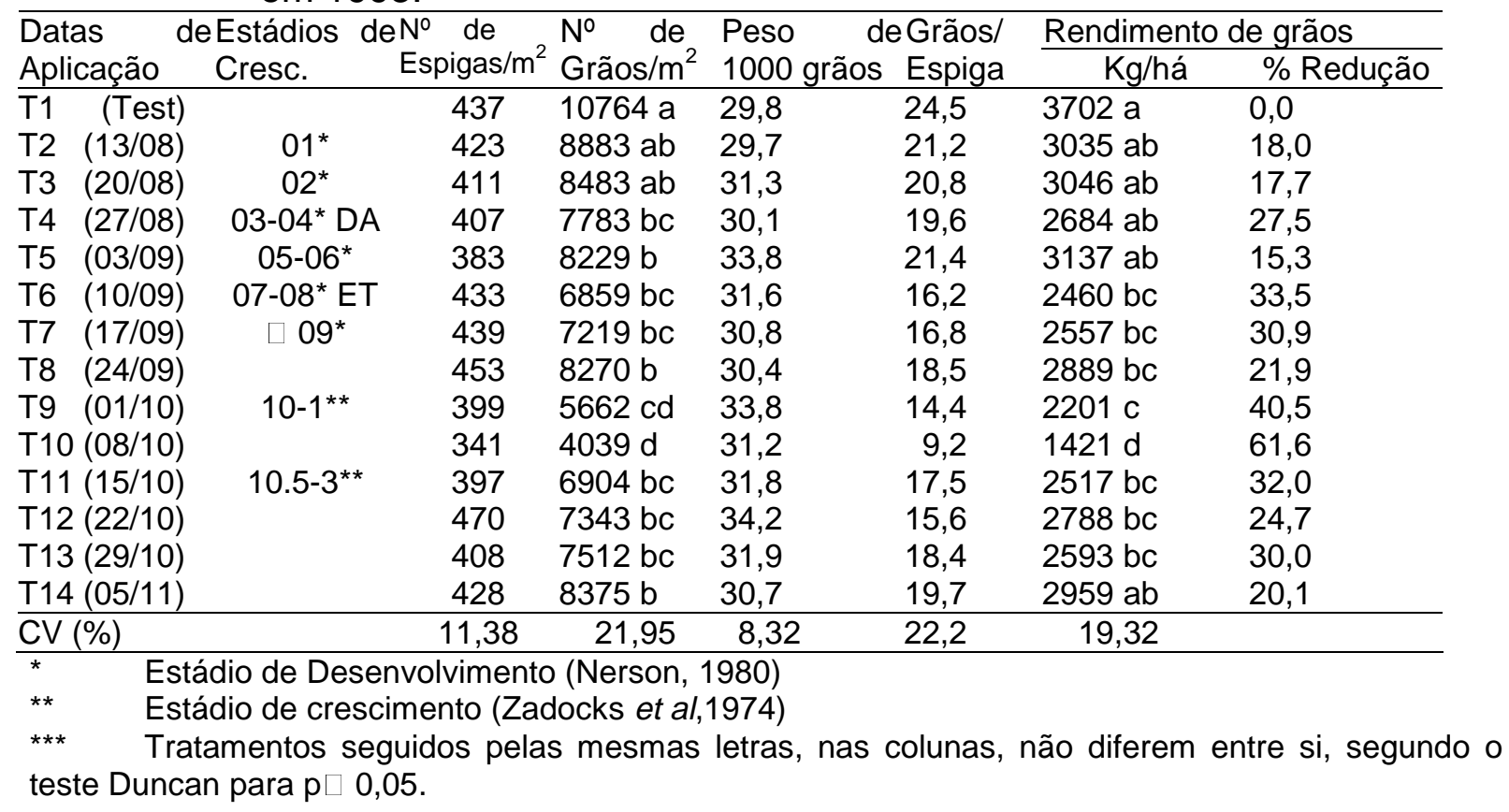


Tabela 05 - Aplicação de herbicida hormonal em diferentes estádios de crescimento de trigo (Embrapa 16) no rendimento de grãos e seus componentes, em 1993.

\begin{tabular}{|c|c|c|c|c|c|c|c|}
\hline Datas & deEstádios de & le № de & $\mathrm{de}$ & Peso & eGrãos/ & Rendiment & le grãos \\
\hline Aplicação & Cresc. & Espigas $/ \mathrm{m}^{2}$ & Grãos $/ \mathrm{m}^{2}$ & 1000 grãos & Espigas & $\mathrm{Kg} / \mathrm{há}$ & $\%$ Red. \\
\hline $\begin{array}{ll}\text { T1 } & \text { (Test }\end{array}$ & & 447 & $10307 \mathrm{abc}$ & $30,9 \mathrm{c}$ & $23,1 \mathrm{abcd}$ & $3661 \mathrm{abc}$ & 0,0 \\
\hline T2 $(23 / 07$ & & 445 & $9927 \mathrm{abc}$ & $32,4 \mathrm{c}$ & $22,4 \mathrm{abcd}$ & $3612 a b c$ & 0,0 \\
\hline T3 $(03 / 08$ & & 476 & $10951 \mathrm{ab}$ & $30,1 \mathrm{c}$ & $23,1 \mathrm{abcd}$ & $3786 a b$ & 0,0 \\
\hline $\mathrm{T} 4 \quad(05 / 08)$ & & 431 & $11370 \mathrm{a}$ & $30,2 \mathrm{c}$ & $26,6 \mathrm{a}$ & $3957 \mathrm{a}$ & 0,0 \\
\hline T5 $(12 / 08)$ & $02^{*}$ & 410 & $9689 \mathrm{bcd}$ & $44,0 \mathrm{a}$ & $23,8 \mathrm{ab}$ & $3780 \mathrm{ab}$ & 0,0 \\
\hline T6 (19/08) & $03-04^{*} \mathrm{DA}$ & 455 & $10685 a b$ & $43,0 \mathrm{a}$ & $23,6 \mathrm{abc}$ & $3689 a b c$ & 0,0 \\
\hline T7 (27/08) & $05-06^{*}$ & 437 & $10663 a b$ & $44,3 \mathrm{a}$ & $24,4 a b$ & $3706 \mathrm{ab}$ & 0,0 \\
\hline T8 $(02 / 09$ & $07-08^{*} \mathrm{ET}$ & 495 & $10807 \mathrm{ab}$ & $43,2 \mathrm{a}$ & $22,1 \mathrm{bcd}$ & $3862 a b$ & 0,0 \\
\hline T9 $(09 / 09)$ & $\square 09^{*}$ & 470 & $10415 \mathrm{abc}$ & $42,4 \mathrm{a}$ & $22,9 \mathrm{abcd}$ & $3517 b c$ & 3,93 \\
\hline T10 (17/09) & & 444 & $8426 d$ & $35,9 \mathrm{bc}$ & $18,9 \mathrm{~d}$ & $2923 d$ & 20,2 \\
\hline T11 $(24 / 09)$ & & 470 & $9003 \mathrm{~cd}$ & $40,9 \mathrm{ab}$ & $19,35 \mathrm{~cd}$ & $3342 \mathrm{c}$ & 8,7 \\
\hline T12 (01/10) & $10-1^{* *}$ & 437 & $3599 \mathrm{f}$ & $19,3 d$ & $8,23 \mathrm{e}$ & $1332 \mathrm{f}$ & 64,2 \\
\hline $\mathrm{T} 13(08 / 10)$ & $10.5-3^{* *}$ & 467 & $6322 \mathrm{e}$ & $31,4 \mathrm{c}$ & $13,6 \mathrm{f}$ & $2350 \mathrm{e}$ & 35,8 \\
\hline T14 (15/10) & $11.1^{\text {** }}$ & 516 & $10632 a b$ & $39,8 \mathrm{ab}$ & $21,3 \mathrm{bcd}$ & $3727 a b$ & 0,0 \\
\hline CV (\%) & & 12,92 & 10,94 & 10,0 & 14,32 & 7,27 & \\
\hline . & & olvimen & Nerson & 80) & & & \\
\hline$* *$ & & ento $(Z$ & ot & 974) & & & \\
\hline$\star * *$ & 0 & $\begin{array}{l}\text { guidos pe } \\
05 \text {. }\end{array}$ & & atras & & & \\
\hline
\end{tabular}

Com relação aos componentes de rendimento, observou-se nos dois anos de estudos (1992 e 1993), nas duas cultivares de trigo, que o número de espigas por metro quadrado não foi afetado por nenhum tratamento (Tabelas 03-04 e 05). Por outro lado, a redução de rendimento de grãos, decorrente da aplicação após o período de tolerância ao herbicida (pós espigueta terminal) observada nos dois anos de estudos, foi decorrente da redução do número de grãos por espiga, com reflexo no número de grãos $/ \mathrm{m}^{2}$, uma vez que não ocorreu redução no número de espigas. O comportamento da cultivar Trigo BR 23, quanto ao peso de mil sementes foi diferente da cultivar Embrapa-16. A cultivar BR 23 em 1993 não apresentou diferença significativa entre os tratamentos. Porém, em 1992 os tratamentos com aplicação de herbicida em torno da antese provocaram aumento no peso de grão (Tabela 03), comparativamente ao controle. Esse aumento no peso de grãos observado, pode ser oriundo da compensação provocada pela redução no número de grãos por espigas. Na cultivar Embrapa-16, quando o herbicida foi aplicado no período de tolerância (Duplo Anel - Espigueta Terminal), ocorreu um aumento no peso de grãos comparativamente ao controle(Tabela 04).

Com relação ao número de espigas por área, a aplicação de herbicida hormonal não provocou efeito significativo nesse parâmetro, nas cultivares estudadas. $\mathrm{O}$ componente que esteve mais associado significativamente com 0 rendimento de grãos foi o número de grão por espiga, com os seguintes coeficientes de correlação: $r=0,93$ para Embrapa-16 e $r=0,93, r=0,91$ para BR 23 em $1992 \mathrm{e}$ 1993, respectivamente.

A duração dos subperíodos para uma mesma cultivar (Trigo BR 23), varia com época de semeadura (Figura 04). Essa variação está associada a variação da temperatura para essa cultivar. A temperatura tem sido considerada o principal fator de ambiente que determina a taxa de desenvolvimento, possivelmente porque todas as plantas e processos de desenvolvimento são sensíveis a ela (Slafer \& 
Rawson,1994). Assim, a diferença de duração dos subperíodos observada na cultivar BR 23 nos dois anos de estudos, pode ser atribuída ao aumento da temperatura acelerando o desenvolvimento. Resultados nesse sentido têm sido freqüentemente reportados (Angus et al., 1981; Slafer \& Savin,1991, Rodrigues et al.,1994). A duração do período considerado tolerante ao herbicida (DA-ET) foi de 11 e 14 dias em 1992 e 1993, respectivamente. Esse comportamento caracteriza a importância da identificação dos respectivos estádios de desenvolvimento das plantas, para o manejo correto do herbicida independente do tempo cronológico.

A duração dos subperíodos também variou com a cultivar (Figura 05). Comparativamente a cultivar BR 23, a cultivar Embrapa 16 apresentou um maior subperíodo de tolerância ao herbicida (DA-ET). Ainda, em 1993 o subperíodo entre a emergência e o duplo anel foi de 22 dias mais longo na cultivar Embrapa 16, comparativamente a BR 23. Dessa forma cuidados devem ser tomados na identificação precisa do período tolerante ao herbicida para se evitar aplicações muito precoces e, consequentemente, danos morfofisiológico na cultura. Esse risco, como pode ser observado na Figura 5, é grande principalmente quando se está manejando cultivares diferentes, como é o caso.

Os resultados permitem afirmar que A Alicação de herbicidas hormonais após o subperíodo Duplo-Anel (DA) - Espigueta Terminal (ET) causam reduções significativas no rendimento de grãos. O subperíodo entre DA-ET, varia com as condições de ambiente e da cultivar utilizada. $O$ número de grãos/espiga é 0 principal componente determinante da redução do rendimento de grãos, quando as aplicações de herbicidas hormonais são realizadas após o estádio de espigueta terminal, em ambas as cultivares de trigo estudadas.

\section{LITERATURA CITADA}

ANDERSEN, S. Environmental factors affecting the response of barley to growth regulator. In: BRITISH WEED CONFERENCE, 2., 1954, Proceedings..2nd, p. 395399, 1954.

ANGUS, J. F. et al. Phasic development in field crops. I. Thermal response in the seedling phase. Field Crops Res., Amsterdam, v. 3, p. 365-378, 1981.

DERSCHEID, L. S. Physiological and morphological responses of barley to 2,4dichorophenoxyacetic acid. Plant Physiol., Bethesda, v. 27, p. 121-134, 1951.

FEEKS, W. De tarwe enthaasmilien. Vers. XVII Tech. Tarwe Comn.Gronigen, $p$. 560-561, 1941.

FRIESEN, G.; OLSON, P. J. The effect of 2,4-D on the development processes in barley and oats. Can. J. Agric. Sci., Ontario, v. 33, p. 315-329, 1953.

FRIESEN, H. A.; BAENZIGER, H.; KEYS, C. H. Morphological and cytological effects of dicamba on wheat and barley. Can. J. Plant Sci., Ottawa, v. 44, p. 288-294,1963.

IVANY, J. A.; NASS, H. G. Effect of herbicides on seedling growth, head deformation and grain yield of spring wheat cultivars. Can. J. Plant Sci., Ottawa, v. 64, p. 25-30, 1984.

LARGE, E. C. Growth stages in cereal - Ilustration of the feekes scale. Plant Pathol., London, v. 3, p. 128-129, 1954. 
LEADEN, M. I.; LOZANO, C. M. Efecto de herbicidas hormanales aplicados em diferentes estados de crescimento del trigo. In: CONGRESO NACIONAL DE TRIGO, 1., 1986, Pergamino. Anais... Pergamino: Asociacion de Ingenieros Agronomos de la Zona Norte de la Provincia de Buenos Aires, [1986]. v. 4, p. 66-73.

LEADEN, M. I.; LOZANO, C. M. Efecto de los momentos de aplicación de herbicidas el rendimento y la selectivadade de dos cultivares de trigo. In: CONGRESO NACIONAL DE TRIGO, VI.; SIMPOSIO NACIONAL DE CULTIVOS DE SIEMBRA OTOÑO-INVERNAL, Bahia Blanca, Argentina. Universidad Nacional del Sur Departamento de Agronomía, v. 4, p. 233-234,2004.

LOZANO, C. M. et al. Relación entre los estados de desarrollos apical y características morfológicas en plantas de trigo. Rev. Fac. Agron., Buenos Aires, v. 5, n. 1/2, p. 51-63, 1984.

NERSON, H.; SIBONY, M.; PINTHUS, J. M. A scale for the assessment of the developmental stages of the wheat (Triticum aestivum L.) spike. Ann. Bot., London, v. 45, p. 203-204, 1980.

PINTHUS, J. M.; NATOWITZ, Y. Response of spring wheat to the application of 2,4-

$D$ at various growth stages. Weed Res., Oxford, v. 7, p. 95-101, 1967.

REUNIÃO DA COMISSÃO SUL-BRASILEIRA DE PESQUISA DE TRIGO, 28., 1996, Passo Fundo. Recomendações... Passo Fundo: Comissão Sul-Brasileira de Pesquisa de Trigo, 1996. 76 p.

RODRIGUES, O. et al. A determinação quantitativa das relações entre temperatura e desenvolvimento em trigo. In: REUNIÃO NACIONAL DE PESQUISA DE TRIGO, 17. 1994, Passo Fundo. Resumos... Passo Fundo: EMBRAPA-CNPT, 1994. p. 55.

SHARMAN, B. C. Morphognesis of 2-4-D induced abnormalities of the inflorescence of bread wheat (Triticum aestivum, L.). Ann. Bot., London, v. 42, p. 145-153, 1978.

SLAFER, G. A.; RAWSON, H. M. Sensitivity of wheat Phasic development to major environmental factors: a re-examination of some assumptions made by physiologists and modellers. Aust. J. Plant Physiol., Victoria, v. 21, p. 393-426,1994.

SLAFER, G. A.; SAVIN, R. Developmental base temperature in different phenological phases of wheat (Triticum estivum L.). J. Exp. Bot., London, v. 42, p. 1077-1082, 1991.

TOTTMAN, D. R. The identification of growth stages in winter wheat with reference to the aplication of growth regulator herbicides. Ann. Appl. Biol., Cambridge, v. 87, p. 213-224, 1977.

TOTTMAN, D. R. Spray timing and the identification of cereal growth stages. In: BRITISH CROP PROTECTION CONFERENCE WEEDS, 24: p. 971-799, 1976

TOTTMAN, D. R.; DUVAL, A. Leaf sheath length as a guide to apical development and spray timing in winter wheat. In: BRITISH CROP PROTECTION CONFERENCE WEEDS, 26, p. 143-149, 1978

TOTTMAN, D. R.; MAKEPEACE, R. J. Na explanation of the decimal code for the growth stages of cereals, with illustrations. Ann. Appl. Biol., Cambridge, v. 93, p. 221-224, 1979.

ZADOCKS, J. C.; GHANG, T. T.; KONZAK, C. F. A decimal code for the growth stages of cereals. Weed Res., Oxford, v. 14, p. 415-421, 1974. 
Figura 4- Duração dos subperíodos da cultivar BR 23 , semeadas em diferentes datas nos anos de 1992 e 1993.

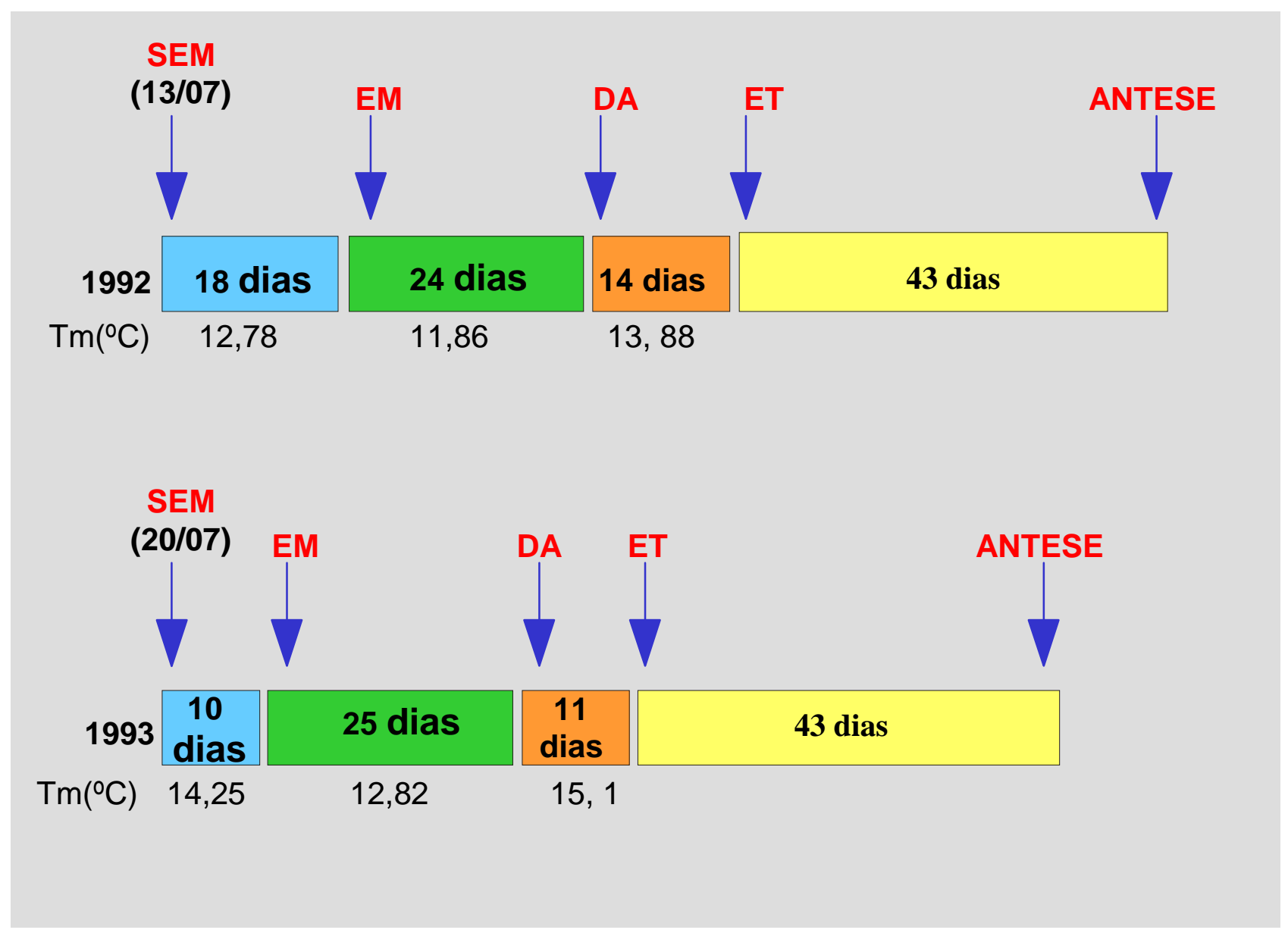


Figura 5- Duração dos subperíodos das cultivares BR 23 e Embrapa 16, semeadas em diferentes datas em 1993
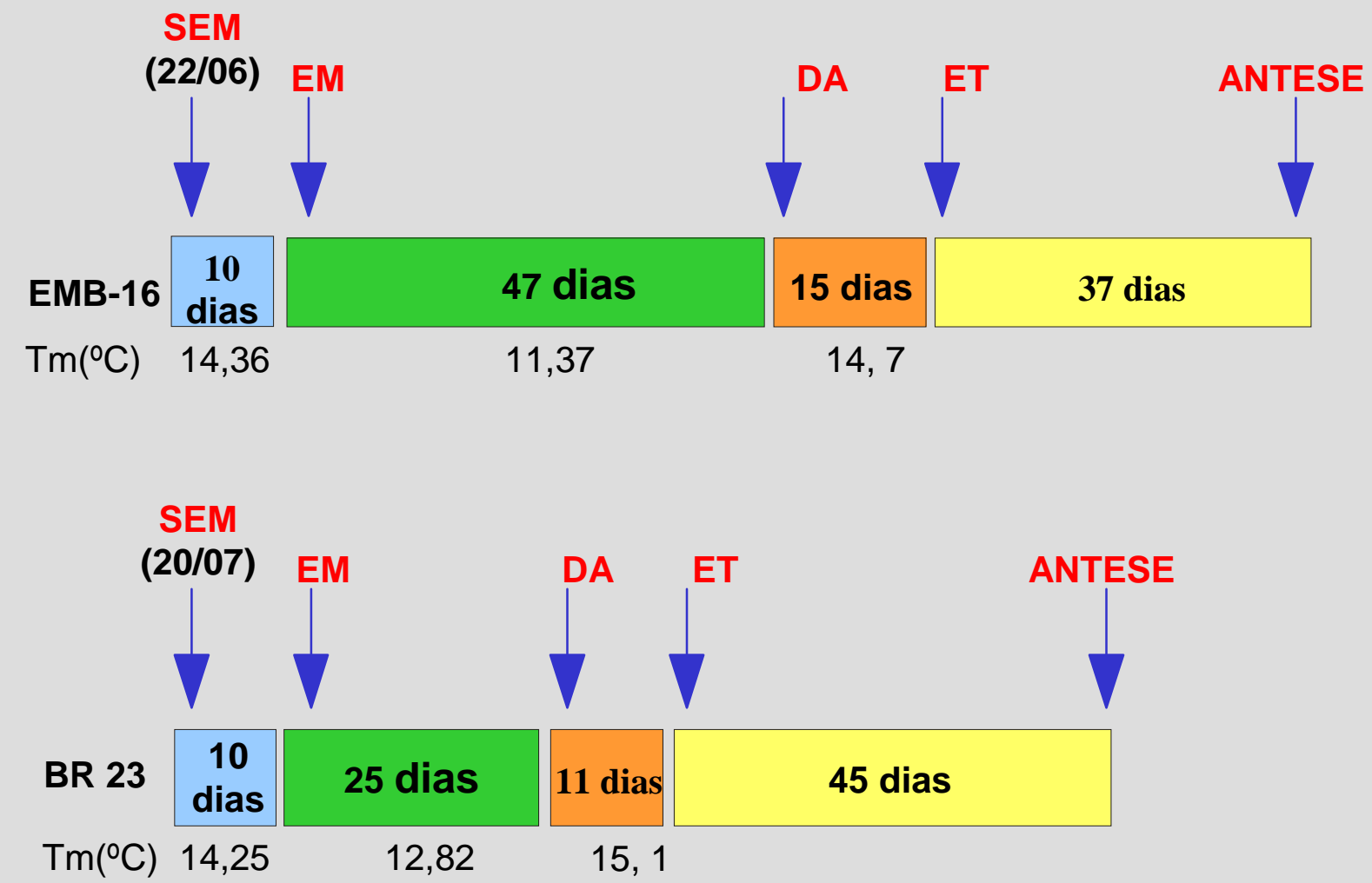